Revista Ingeniería y Región. 2016;16(2): 65-71

http://dx.doi.org/10.25054/22161325.1300

\title{
Estrategias para estimar el coeficiente de uniformidad en laterales de riego
}

\author{
Strategies to estimate uniformity coefficient \\ in irrigation laterals
}

Marlio Bedoya Cardoso ${ }^{1}$ y Vicente Ángeles Montiel $^{2}$

\begin{abstract}
Resumen
Las pérdidas por fricción y localizadas, el espaciamiento entre emisores, la pendiente del terreno, la velocidad de descarga y el régimen del emisor, son parámetros importantes para determinar la uniformidad de Riego (aplicación). Por lo anterior, el presente trabajo se realizó con el fin de estimar la uniformidad de aplicación, proponiendo estrategias alternativas de aforo de emisores en laterales que tiendan a reducir el número de lecturas. Las pruebas se realizaron en un lateral de 50 metros $(\mathrm{m})$ de longitud y 13.5 milímetros de diámetro interior, el lateral se trabajó con espaciamiento constante de $0.40 \mathrm{~m}$. Todos los goteros de los laterales se aforaron 3 veces para determinar su promedio. Los resultados indican que la propuesta de aforar goteros a (25-50-75 y 20-40-80) \% de la longitud del lateral, predicen bien la uniformidad de aplicación, con un nivel de significancia del $95 \%$ en la prueba de hipótesis de varianza.
\end{abstract}

Palabras clave: uniformidad de aplicación; pérdidas por fricción; pérdidas localizadas.

\begin{abstract}
Friction and localized losses, the spacing between emitters, the slope of the ground, the emitter flow rate and flow regimen, are important parameters to determine the irrigation uniformity (application). Accordingly, the present study was carried out in order to predict the uniformity of application and to propose alternative strategies for the measurement of lateral emitters that tend to reduce the number of readings. The tests were conducted in one lateral of 50 meters $(\mathrm{m})$ length and 13.5 millimeters interior diameter. In this lateral, a constant spacing of $0.40 \mathrm{~m}$ was used. The results indicate that the proposed lateral's longitude of measuring drippers (25-50-75 and 20-40$80) \%$ predicts good uniformity of application with a significant level of $95 \%$ in the hypothetical test of variance.
\end{abstract}

Keywords: emission uniformity; friction losses; localized losses.

1 Estudiante de Maestría en Ingeniería Agrícola y Uso integral del agua. Universidad Autónoma Chapingo. Carretera. México-TexcocoKm. 38.5. marlio.bedoya@gmail.com

2 Doctor en Ingeniería de Irrigación. Universidad Autónoma Chapingo. Carretera. México-Texcoco- Km. 38.5. vangelesm@msn.com

Recibido: 12 de marzo de 2016 • Aprobado: 13 de octubre de 2016. 


\section{Introducción}

La pérdida de carga en tuberías con salidas múltiples igualmente espaciadas, será de menor magnitud que la pérdida de carga en una tubería similar que trasmite el gasto completo sobre toda su longitud debido a que el gasto se disminuye establemente cada vez que pasa por una salida (García y Briones, 2007). Una tubería con salidas múltiples es una estructura hidráulica, cuyo diseño está limitado por la presión de entrada, la topografía del terreno y por la uniformidad de aplicación de agua que se ve afectada por la pérdida total de energía a lo largo de la tubería, así como las características hidráulicas de la salida (Yildirim, 2006).

La determinación exacta de las pérdidas por fricción en una tubería con salidas múltiples requiere de un análisis tramo por tramo a partir de la última salida, $\mathrm{y}$ para evitar este incómodo análisis, algunos investigadores han propuesto expresiones que agilizan dicho proceso, entre ellos se destacan: (Christiansen, 1942); (Scaloppi, 1988); (Anwar, 1999a); (Anwar, 1999b); (Chineas y Rodríguez, 2006); y el más reciente (Ángeles, et al., 2009).

Las pérdidas totales (fricción y menores) y el espaciamiento entre emisores afectan la distribución de agua en un lateral de riego, por ello, se han desarrollado expresiones numéricas que sirven como índice para asegurar una uniformidad y con este propósito se utilizan expresiones llamadas coeficientes de uniformidad, los cuales son expresado en porcentaje y han sido trabajados por (Christiansen, 1942); (Keller y Karmeli, 1974); (Keller y Karmeli 1975); (Barragán, et al., 2005).

\section{Metodología}

El presente trabajo se realizó en las instalaciones del laboratorio de ingeniería de riego campo: Tlapeaxco, Montecillo y la Cerona, de la Universidad Autónoma Chapingo, ubicado en el km. 38 carretera México Texcoco, con las siguientes coordenadas $19^{\circ} 29^{\prime}$ latitud Norte, $98^{\circ} 54^{\prime}$ de latitud Oeste a una altura de 2250 m.s.n.m. (Figura 1).

\section{Determinación de la presión del lateral}

La presión de operación fue $1.83 \mathrm{~kg} \mathrm{~cm}^{-2}$ (26 PSI), con la cual se asegura que al final de este lateral llegara la presión de trabajo del gotero de $8 \mathrm{l} / \mathrm{h}$, ya que el rango de operación de estos goteros es de 0.49 a 4.22 $\mathrm{kg} \mathrm{cm}^{-2}$ (7-60 PSI), esta presión de operación se determinó con la siguiente expresión:

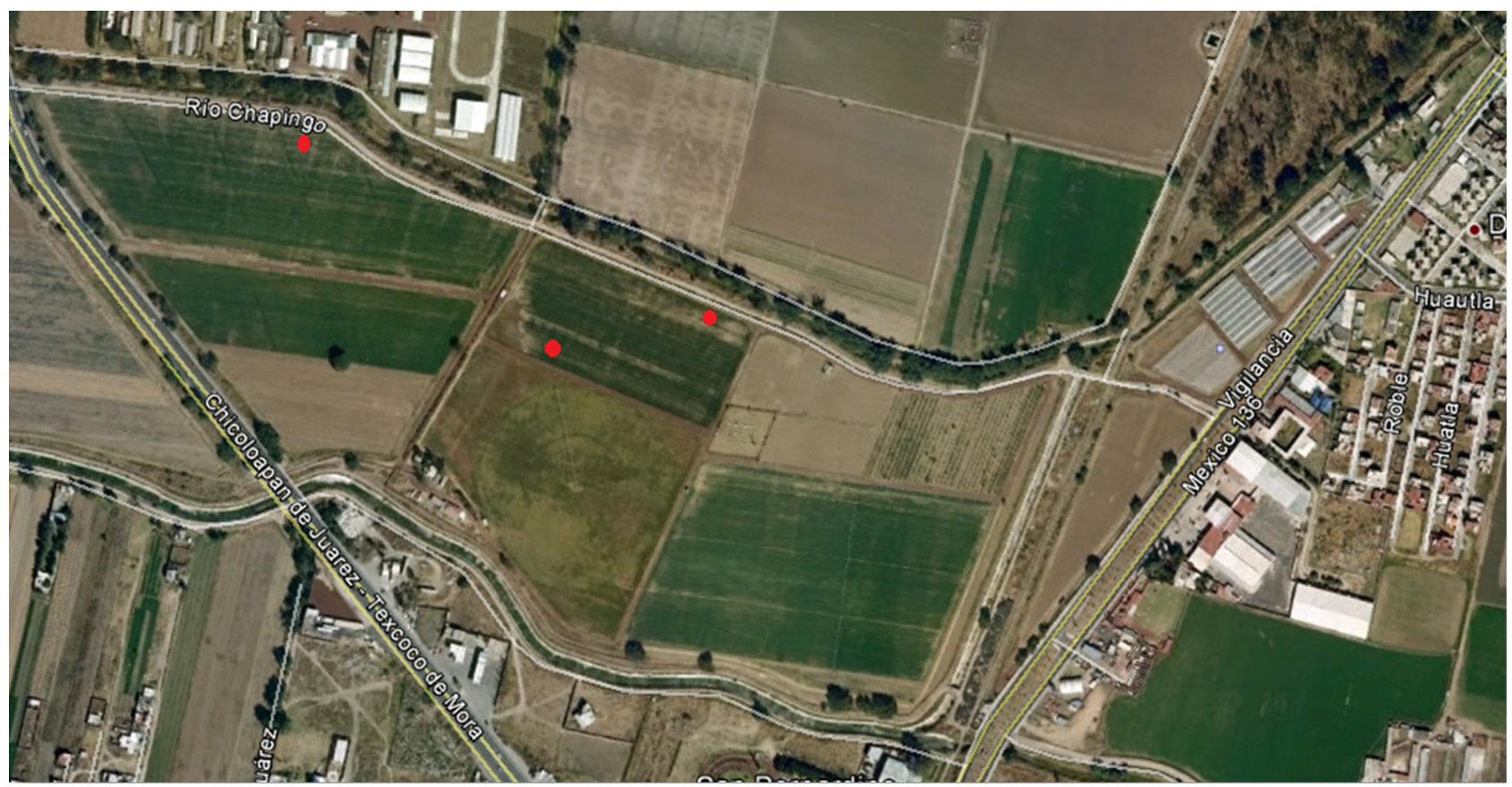

Figura 1. Vista satelital del campo experimental Tlapeaxco, Montecillo y la Cerona. Google Earth $2012 \AA$ 


$$
h_{l}=h_{o}+\left(\frac{3}{4}\right) h_{f}^{*}
$$

Donde:

$h_{l}$ es presión a la estrada del lateral, en m $h_{o}$ es la presión de operación del emisor, en m y $h_{f} *$ son las pérdidas por fricción corregidas de la tubería, en $\mathrm{m}$.

Las pérdidas por fricción se calcularon utilizando una relación matemática entre la ecuación de DarcyWeisbach y Blasius, la cual es afectada por la temperatura del agua y a $18^{\circ} \mathrm{C}$ esta relación es la siguiente:

$$
h_{f}=0.000798 * \frac{Q^{1.75}}{D^{4.75}} * L(2)
$$

Donde:

$h_{f}=$ Pérdidas por fricción, $m$

$Q=$ es el caudal total del lateral, en $\mathrm{m}^{3} / \mathrm{s}$

$D=$ es el diámetro de la tubería, en $m y$

$L=$ es la longitud del lateral, en $m$

Con la ecuación (2) se determinan las pérdidas por fricción en una tubería cerrada, pero para convertirlas a pérdidas en tuberías con salidas múltiples se multiplican con el factor de (Christiansen, 1942) expresado de la siguiente forma:

$$
F=\frac{1}{m+1}+\frac{1}{2 N}+\frac{(m-1)^{0.5}}{6 N^{2}}
$$

Donde:

$F=$ es el factor de Christiansen;

$M=$ es el exponente de la velocidad en la fórmula usada para calcular la pérdida de presión causada por la fricción

$N=$ es el número de salidas a lo largo de la tubería.

Las pérdidas por conexión (menores) se expresaron en longitud equivalente utilizando la ecuación de (Keller y Bliesner, 1990).

$$
J=\left(\frac{s e+f e}{s e}\right)(4)
$$

Donde:

$J=$ es la pérdida por conexión (m);

$s_{e}=$ es la separación entre emisores (m) y

$f_{e}=$ es la longitud equivalente de la pérdida de carga por conexión (m), cuyo valor es $0.15 \mathrm{~m}$
Las pérdidas totales en la tubería o pérdidas por fricción corregidas por la conexión del emisor están dadas por la siguiente expresión:

$$
h_{f}^{*}=h_{f} * F * J(5)
$$

\section{Estimación del coeficiente de Uniformidad}

Una vez fijada la presión de operación se hizo el respectivo aforo volumétrico con la ayuda de probetas graduadas y un cronómetro para establecer la cantidad de agua que se estaba suministrando al lateral con dicha presión, éste procedimiento se hizo tres veces, luego se conectó el lateral con espaciamiento constante de $0.40 \mathrm{~m}$, en el cual se aforaron los 125 goteros 3 veces para determinar su promedio. La presión de operación en el lateral se verificó y ajustó cada diez metros cuando ya se habían aforado por lo menos 25 goteros para asegurar la misma presión de operación en toda la medición.

Después de generados los resultados en el lateral, se determina el coeficiente de uniformidad (aplicación), utilizando las ecuaciones de (Keller y Karmeli, 1974); (Keller y Karmeli, 1975).

$$
\begin{gathered}
C_{u}=100 *\left[1-u+u * \frac{q_{25 \% h}}{q_{\text {medh }}}\right] * \frac{q_{\text {mind }}}{q_{\text {medd }}} \\
C_{u}=100 \frac{q_{25 \% h}}{q_{\text {medd }}}(7) \\
C_{u}=100 *\left[1-\frac{1.27 * C v f}{\sqrt{e p}}\right] * \frac{q_{\text {mind }}}{q_{\text {medd }}}(8)
\end{gathered}
$$

Donde:

$C_{u}=$ es el coeficiente de uniformidad (aplicación) $q_{25 \% h}=$ es el caudal medio del $25 \%$ de los emisores de menor caudal $(l / h)$,

$q_{\text {medh }}=$ es el caudal medio de la muestra de emisores operados a presión de referencia $(l / h)$

$q_{\text {mind }}=$ es el caudal mínimo $(l / h)$

qmedd = es el caudal medio de dimensionamiento $o$ diseño $(l / h)$

$c v f=$ es el coeficiente de variación de fabricación y ep $=$ es el número de emisores por planta.

Además de las anteriores ecuaciones se utiliza la expresión de coeficiente de uniformidad propuesto por (Barragán et al., 2005).

$$
C_{u}=1-\sqrt{\left(1-\frac{q \operatorname{mind}}{q m e d d}\right)^{2}+\left(\frac{1.27 * C v f}{\sqrt{e p}}\right)^{2}}
$$




\section{Método para propuesta de aforo}

Para predecir la uniformidad de aplicación en laterales de riego con emisores autocompensados, sin la necesidad de aforar todos los goteros, se estableció el tamaño de muestra con la expresión que se describen a continuación:

$$
n=\frac{N * Z^{2} * S^{2}}{d^{2} *(N-1)+\left(Z^{2} * S^{2}\right)}
$$

Dónde:

$N=$ es el número total de emisores

$Z=$ es la seguridad del 95\% con coeficiente de 1.96

$S^{2}=$ es la varianza $y$

$d=$ es el error esperado $0.13(\mathrm{l} / \mathrm{h})$.

Luego de fijado el número de emisores a medir por la ecuación 10 , se diseñaron cuatro procedimientos para seleccionar los goteros a medir a lo largo del lateral, estos procedimientos se describen a continuación:

1. Se generaron números aleatorios del 1 al 125 , de 50 muestras de tamaño $\mathrm{n}=5,10,15,20,25,30$, para el lateral.

2. En este procedimiento, se divide el lateral en 4 partes iguales para realizar los aforos en tres sitios ubicados a 1/4, 1/2, y $3 / 4$ de la longitud del lateral, cada sitio sirvió como punto medio, ya que, se aforaron emisores antes y después de dicho punto.

3. Se decide aforar los goteros que se encuentran a $(20,40$ y 80$) \%$ de la longitud del lateral.

4. En el cuarto y último procedimiento se resuelve aforar los goteros ubicados a $(20,40$ y 60$) \%$ de la longitud del lateral.

\section{Procedimiento para el análisis de resultados}

Los datos arrojados en este trabajo fueron tabulados, analizados y graficados con la ayuda de una hoja de cálculos de Excel del programa de Microsoft 2010, en la cual se determinaron los promedios, varianzas y coeficientes de uniformidad, así mismo se realizó la prueba de hipótesis de varianza con nivel de significancia del $95 \%$ para determinar si presentaban evidencias relevantes.

\section{Resultados}

\section{Presión de operación en el lateral}

La pérdida por fricción en tuberías con salidas múltiples fue $6.86 \mathrm{~m}$ y se calculó utilizando las ecuaciones 2 y 3 , la cual, luego se corrigieron con el valor de 1.34 (Ecuación 4), cuyo valor representa la pérdida generada por conexión de emisores. Con las pérdidas por fricción corregidas y presión de operación del emisor $\left(1 \mathrm{~kg} \mathrm{~cm}^{-2} ; 10 \mathrm{~m}\right)$, se estableció la presión al inicio del lateral utilizando la ecuación 1.

\section{Determinación del coeficiente de uniformidad}

En el Tabla 1 se pueden ver los resultados de los coeficientes de uniformidad del lateral con dos repeticiones utilizando las ecuaciones $6,7,8$ y 9 , en ella se observa que el coeficiente de uniformidad en la segunda repetición no difiere en más de $3 \%$ en casi todas las ecuaciones.

Tabla 1. Coeficientes de Uniformidad (CU) en el lateral con dos repeticiones

\begin{tabular}{l|c|c}
\hline $\begin{array}{c}\text { Ecuaciones de coeficientes } \\
\text { de uniformidad }\end{array}$ & $\boldsymbol{L . T}^{\mathbf{1}} \boldsymbol{\%}$ & $\boldsymbol{L} . \boldsymbol{T}^{\mathbf{2} \%}$ \\
\hline Ecuación 6 & 75.35 & 77.91 \\
\hline Ecuación 7 & 93.94 & 93.87 \\
\hline Ecuación 8 & 77.76 & 80.46 \\
\hline Ecuación 9 & 79.97 & 82.72 \\
\hline
\end{tabular}

1. Coeficiente de uniformidad en el lateral tradicional primera prueba.

2. Coeficiente de uniformidad en el lateral tradicional segunda repetición.

\section{Propuestas de aforo primer procedimiento}

En las figuras 2 y 3 se observa el comportamiento de los diferentes coeficientes de uniformidad, con 50 muestras aleatorias de tamaño $\mathrm{n}=5$ y $\mathrm{n}=25$ respectivamente, en ellas se puede afirmar que su utilización es inexacta al momento de predecir la uniformidad, ya que la probabilidad de encontrar errores inferiores al $5 \%$ es de tan solo el $18 \%$ en todas las muestras de tamaño 5 y 25 . Sin embargo, a mayor tamaño de la muestra el coeficiente de uniformidad se acerca al real. 


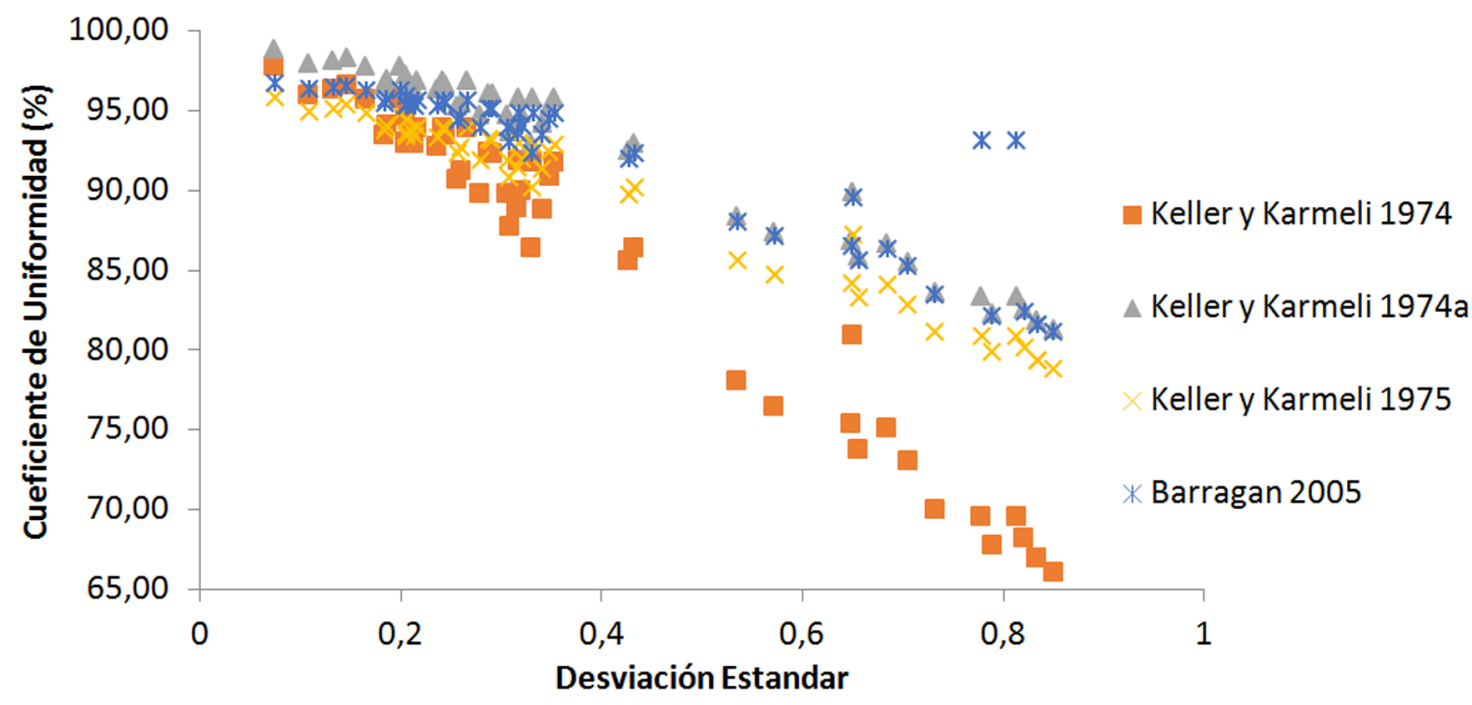

Figura 2. Comportamientos de los coeficientes de uniformidad con $n=5$.

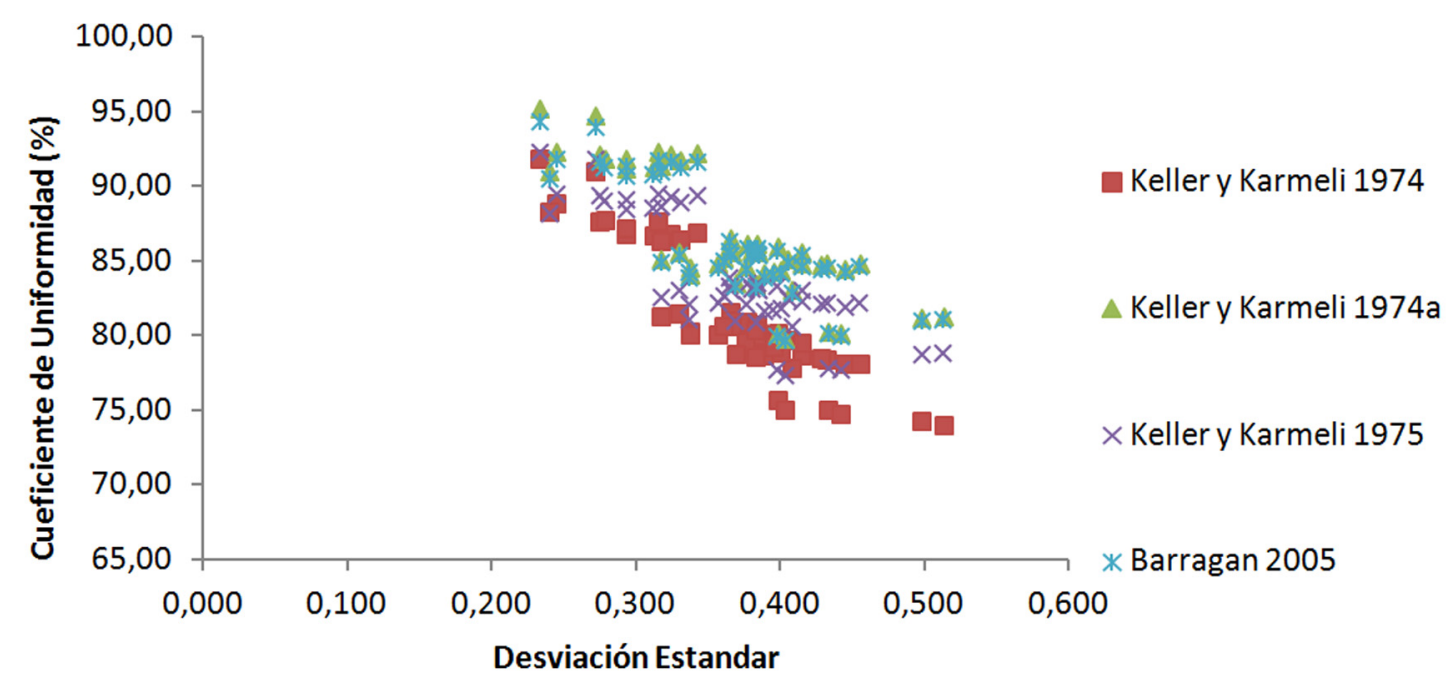

Figura 3. Comportamientos de los coeficientes de uniformidad con $n=25$.

Propuesta de aforo segundo y tercer procedimiento

La utilización del segundo y tercer procedimiento para predecir la uniformidad de aplicación en el lateral sin necesidad de aforar todos los goteros, con dos repeticiones generó errores inferiores al 4.2 y $3.7 \%$ respectivamente (Tabla 2).

Para establecer si los errores en predicción de la uniformidad de aplicación con el segundo y tercer procedimiento son relevantes, se realizó una prueba de hipótesis de varianzas, en ella se comprobó con un nivel de significancia del 95\% que no existen evidencias significativas entre los procedimientos de aforo a (25-50-75 y 20-40-80) \% de la longitud del lateral, lo que indica que las varianzas en estos dos procedimientos son iguales a la del lateral evaluado.

\section{Propuesta de aforo cuarto procedimiento.}

Puede verse en el cuadro 3, que la utilización de este procedimiento de aforo a (20-40-60) \% de la longitud del lateral, generó errores inferiores al 14.8\% en el lateral. Este error del $14.8 \%$ en la estimación de la uniformidad, mostró evidencias importantes en la prueba de hipótesis de varianza con un nivel de significancia del $95 \%$, lo cual indica que la hipótesis nula de varianzas iguales se rechaza. 
Tabla 2. Estimación de la Uniformidad con los procedimientos de aforo en el lateral

\begin{tabular}{|c|c|c|c|c|c|}
\hline & \multicolumn{5}{|c|}{ Lateral espaciamiento constante 0.4 metros (Primera repetición) } \\
\hline & Christiansen & $\begin{array}{c}\text { Keller y } \\
\text { Karmeli 1974 } \\
\end{array}$ & $\begin{array}{c}\text { Keller y Karmeli } \\
1974 \text { p. } \\
\end{array}$ & \begin{tabular}{|c|} 
Keller y \\
Karmeli 1975 \\
\end{tabular} & $\begin{array}{c}\text { Barragán } \\
2005 \\
\end{array}$ \\
\hline Real (medición completa) & 96.76 & 75.35 & 93.94 & 77.76 & 79.97 \\
\hline 2 do Procedimiento ${ }^{1}$ & 96.10 & 73.81 & 91.96 & 77.81 & 80.02 \\
\hline 3er Procedimiento ${ }^{2}$ & 97.01 & 79.03 & 94.39 & 81.18 & 83.45 \\
\hline 4to Procedimiento ${ }^{3}$ & 97.60 & 90.06 & 96.29 & 90.68 & 92.84 \\
\hline Error 2do Procedimiento & $0.66 \%$ & $1.54 \%$ & $1.98 \%$ & $0.05 \%$ & $0.05 \%$ \\
\hline Error 3er Procedimiento & $0.25 \%$ & $3.68 \%$ & $0.45 \%$ & $3.42 \%$ & $3.48 \%$ \\
\hline \multirow[t]{3}{*}{ Error 4to Procedimiento } & $0.84 \%$ & $14.71 \%$ & $2.35 \%$ & $12.92 \%$ & $12.87 \%$ \\
\hline & \multicolumn{5}{|c|}{ Lateral espaciamiento constante 0.4 metros (segunda repetición) } \\
\hline & Christiansen & $\begin{array}{c}\text { Keller y } \\
\text { Karmeli } 1974 \\
\end{array}$ & $\begin{array}{c}\text { Keller y Karmeli } \\
1974 \text { p. } \\
\end{array}$ & \begin{tabular}{|c|} 
Keller y \\
Karmeli 1975 \\
\end{tabular} & $\begin{array}{c}\text { Barragán } \\
2005 \\
\end{array}$ \\
\hline Real (medición completa) & 96.67 & 77.91 & 93.87 & 80.46 & 82.72 \\
\hline 2do Procedimiento ${ }^{1}$ & 95.80 & 81.61 & 93.65 & 84.49 & 86.79 \\
\hline 3er Procedimiento ${ }^{2}$ & 96.23 & 78.07 & 93.46 & 80.99 & 83.25 \\
\hline 4to Procedimiento ${ }^{3}$ & 97.15 & 91.97 & 96.53 & 92.37 & 94.38 \\
\hline Error 2do Procedimiento & $0.87 \%$ & $3.70 \%$ & $0.22 \%$ & $4.03 \%$ & $4.07 \%$ \\
\hline Error 3er Procedimiento & $0.44 \%$ & $0.16 \%$ & $0.41 \%$ & $0.53 \%$ & $0.53 \%$ \\
\hline Error 4to Procedimiento & $0.48 \%$ & $14.06 \%$ & $2.66 \%$ & $11.91 \%$ & $11.66 \%$ \\
\hline
\end{tabular}

1 Aforo a 2550 y $75 \%$ de la longitud total

2 Aforo a 2040 y $80 \%$ de la longitud total

3 Aforo a 2040 y $60 \%$ de la longitud total

\section{Conclusión}

Los procedimientos para aforar emisores ubicados a $\operatorname{los} 25,50$ y $75 \%$ y a $\operatorname{los} 20,40$ y $80 \%$ de la longitud del lateral son estrategias prácticas para estimar la uniformidad de aplicación y reducir el número de lecturas en laterales de riego, pues con ellos se generan errores Máximos de $4.07 \%$ y mínimos de $0.05 \%$ con todas las ecuaciones utilizadas, así mismo, la prueba de hipótesis de igualdad de varianza muestra con un nivel de significancia de $95 \%$ que no existen evidencias importantes entre las varianzas de los procedimientos mencionados anteriormente, frente a la varianza de los datos reales en el lateral evaluado.

\section{Referencias bibliográficas}

Ángeles, V., Carrillo, M., Ibáñez, L. A., Arteaga, R. and Vázquez, M.A., 2009. Estrategias alternativas en la determinación del factor de ajuste en el cálculo de la pérdida de carga por fricción en tuberías con salidas múltiples. Ingeniería del agua, 16(1), p.41-50.
Anwar, A. A., 1999. Factor $G$ for pipelines with equally spaced multiple outlets and outflow. Journal of Irrigation and Drainage Engineering, 125(1), p.3438. https://doi.org/10.1061/(ASCE)0733-9437(1999) 125:1(34)

Anwar, A.A., 1999. Adjusted factor $\mathrm{G}$ a for pipelines with multiple outlets and outflow. Journal of irrigation and drainage engineering, 125(6), p.355-359. https: //doi.org/10.1061/(ASCE)0733-9437(1999) 125:6(355)

Barragán, J., Bralts, V. and Wu, I.P., 2006. Assessment of emission uniformity for micro-irrigation design. Biosystems Engineering, 93(1), p.89-97. https:// doi.org/10.1016/j.biosystemseng.2005.09.010

Chineas, R. R. Dominguez, A., 2006. Total friction loss along multiple outlets pipes with open end. Journal of irrigation and drainage engineering, 132(1), p.3140. https://doi.org/10.1061/(ASCE)0733-9437(2006) $132: 1(31)$ 
Christiansen, J. E., 1942. Irrigation by sprinkling (No. 04; USDA, FOLLETO 1532.).

García, C. I., Briones, S. G., 2007. Sistemas de riego: por aspersión y goteo (No. 631.587 G3S5).

Keller, J., Karmeli, D., 1975. Trickle irrigation design (No. 04; TC805, K3.). Glendora, CA: Rain Bird Sprinkler Manufacturing Corporation.

Keller, J. Bliesner, R. D., 1990. Sprinkle and trickle irrigation
Keller, J., Karmeli, D., 1974. Trickle irrigation design parameters. Transactions of the ASAE, 17(4), p.678684. https://doi.org/10.13031/2013.36936

Scalopi, J. E., 1988. Adjusted F factor for multiple-outlet pipes. Journal of Irrigation and Drainage Engineering, 114(1), p.169-174. https://doi.org/10.1061/(ASCE) 0733-9437(1988)114:1(169)

Y1ldırım, G., 2006. Hydraulic analysis and direct design of multiple outlets pipelines laid on flat and sloping lands. Journal of irrigation and drainage engineering, 132(6), p.537-552. https://doi.org/10.1061/(ASCE) 0733-9437(2006)132:6(537) 
